\title{
Discharge flow of granular media from rectangular silos: role of an obstacle and modelling by an orifice at the corner
}

\author{
Samira Laidaoui ${ }^{1,2, *}$, Pascale Aussillous ${ }^{1}$, Mohammed Djermane $^{2}$, and Blanche Dalloz-Dubrujeaud ${ }^{1}$ \\ ${ }^{1}$ Aix-Marseille Univ, CNRS, IUSTI, Marseille, France \\ 2 Tahri Mohammed university of Bechar, FIMAS, BP417, Bechar 08000, Algeria
}

Received: 9 October 2019 / Accepted: 8 June 2020

\begin{abstract}
We present an experimental study on the discharge flow of a granular media from a rectangular silo with a cylindrical obstacle placed above the outlet. As described in the literature, the presence of an obstacle decreases the flow rate, but the characteristic lengths to be chosen in the flow rate law are not known. To predict the flow rate we vary the obstacle diameter and vertical position, the outlet size and the particle diameter. However due to the large number of parameters we find that the characteristic length which controls the flow rate cannot be thoroughly defined. To model the effect of an obstacle on the flow rate we design a new configuration with an orifice at the corner of the silo. We study the two extreme cases (a bottom orifice at the wall and a lateral orifice) and we show that for the corner all the data are in between these two cases.
\end{abstract}

Keywords: Granular flow / silo discharge

\section{Introduction}

Confined geometries like silos are widely used for the processing of granular and powder materials which are important in many engineering applications, but the discharge flow of granular matter in silos remains an open subject of study.

A very famous problem in silos is jamming. A practical solution commonly implemented to solve this problem is the placement of an obstacle just above the outlet. In this regime, it has been shown that the obstacle presence reduces the jamming probability and increase the flow rate $[1-3]$. However few studies are devoted to the study of the flow in the presence of an obstacle in the continuous regime. Most of the works have analysed the effect of using differently shaped inserts on the flow pattern [4-10] but the influence of an obstacle on the discharge flow rate has not been thoroughly studied.

Without obstacle, the discharge flow of a granular media from a rectangular silo, with an outlet of size $D$ spanning the thickness, $W$ (see Fig. 1a), have been widely studied since the pioneer work of Hagen in 1852 [11] and the empirical expression proposed by Beverloo et al. [12]. Recently Janda et al. [13] have studied the flow of monodisperse particles through orifices from a rectangular silo with a single layer of particles in the thickness. They have found that for the whole range of apertures,

\footnotetext{
e-mail: samira.laidaoui@etu.univ-amu.fr
}

the velocity profiles at the outlet are self-similar and the vertical velocity at the centre is given by $v_{0} \propto \sqrt{g D}$ due to an accelerating zone which scale with the size of the orifice [14]. In the same way, the density profile was found to be self similar and the density at the centre $\phi_{0}$ decreases with the outlet size which corresponds to a dilation of the particles at the outlet. Benyamine et al. [15] have shown that this dilation depends on the dimensionless aperture size $D / d_{p}$, where $d_{p}$ is the particle diameter and have proposed an empirical relation $\phi_{0} \propto \phi\left(1-\alpha e^{-\beta D / d_{p}}\right)=\phi G\left(D / d_{p}\right)$ where $\phi$ is the bulk particle volume fraction and $\alpha, \beta$ are fitting parameters. Consequently they have proposed the following expression for the discharge flow rate, $Q$, from a rectangular silo:

$$
Q=C \rho_{p} \phi G\left(\frac{D}{d_{p}}\right) W D \sqrt{g D}
$$

where $g$ is the gravitational acceleration, $\rho_{p}$ the particle density and $C$ a fitting parameter.

Recently Zhou et al. [16] have shown experimentally and using continuous simulation with a frictional rheology that if the outlet is located at a lateral position the silo thickness plays a role due to the confinement of the geometry. Indeed they have shown that the parietal friction tends to rotate the streamlines to align them toward the gravity with an angle at the outlet $\sin \theta_{0} \propto(1+\gamma D / W)^{-1 / 2}$, whereas the norm of the velocity scales with the aperture 
size $U_{0} \propto \sqrt{g D}$. Then for large number of beads in the aperture they predicted that the flow rate follows

$$
Q=C_{l} \rho_{p} \phi W D \frac{\sqrt{g D}}{\sqrt{1+\gamma D / W}}
$$

where $C_{l}$ and $\gamma$ are fitting parameters. Thus they have observed two regimes of flow. The first regime when the aperture ratio $A=D / W$ is small corresponds to the classical Hagen law where $Q \propto W D^{3 / 2}$. The second regime when $A$ is large is controlled by the confinement where $Q \propto W^{3 / 2} D$.

This paper is devoted to the experimental investigation of discharge flow from a rectangular silo in the presence of an obstacle in the continuous regime. The experiments are described in Section 2. Then results for the discharge flow of the silo with an obstacle are shown in Section 3. To simplify the problem a new experimental configuration is proposed in Section 4 where the orifice is placed at the corner of silo without obstacle in order to find out which parameters are relevant to describe the flow rate.

\section{Experiments}

All experiments have been carried out with a rectangular silo with a flat bottom (of height $H=1100 \mathrm{~mm}$, width $L=100 \mathrm{~mm}$ and thickness $W=5 \mathrm{~mm}$ ). As shown in Figure 1, different configurations were designed: outlet of adjustable length $\mathrm{D}$ at the bottom (Fig. 1a), outlet of adjustable length $D$ at the bottom and a cylindrical obstacle of diameter, $D_{\text {obs }}$, and altitude, $h_{0}$, above the outlet (Fig. 1b) and an outlet in the right bottom corner with adjustable horizontal size, $D_{x}$, and vertical size, $D_{y}$ (Fig. 1c). All configurations have a free surface at the top, and the rear and front walls are made of transparent glass plates. Table 1 summarises the different parameters used for this study.

The granular material consists of smooth spherical ceramic beads of density $\rho_{p}=6000 \mathrm{~kg} / \mathrm{m}^{3}$ which are almost monodisperse in diameter with a dispersion of $\pm 20 \%$ (see Tab. 1).

Keeping the outlet of the silo closed, a known weight $m_{p}$ of ceramic beads are poured into the silo from the top. Measuring the height of the granular column, the initial bulk particle volume fraction $\phi$ is calculated for each experiment. Opening the outlet, the grains fall freely out of the silo and are collected in a vessel placed on an electronic balance with a precision of $1 \mathrm{~g}$ (Mettler Toledo $6002 \mathrm{~S}$ ) and the temporal evolution of the mass $m(t)$ is recorded at a frequency of $20 \mathrm{~Hz}$. Each experiment is repeated twice to check the reproducibility of the process. The instantaneous mass flow rate $Q_{i}$ is obtained by measuring the local slope of the temporal evolution (sliding mean value over $1 \mathrm{~s}$ ). Figure 2 shows that in the presence of an obstacle the flow rate remains stationary, the mean flow rate $Q$ is obtained by averaging the instantaneous flow rate over the steady state. Oscillations were observed for all experiments and are due to the response time of the balance.
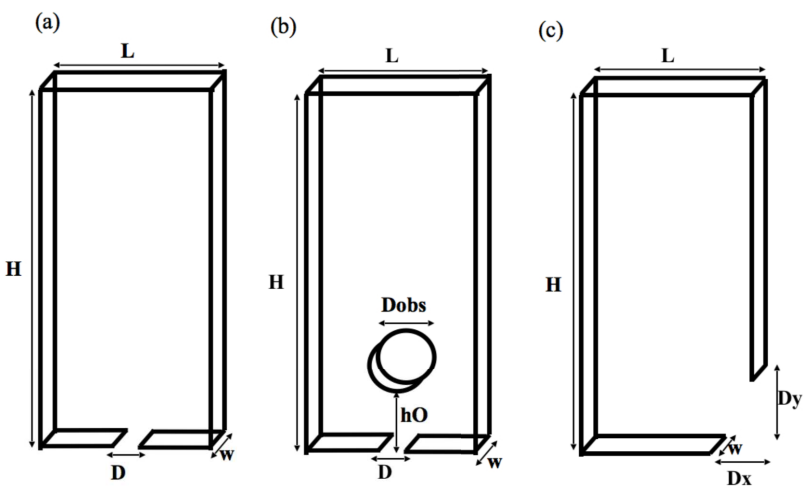

Fig. 1. Different configuration of silo. (a) Outlet at the bottom. (b) Outlet at the bottom with obstacle. (c) Outlet in the corner of silo.

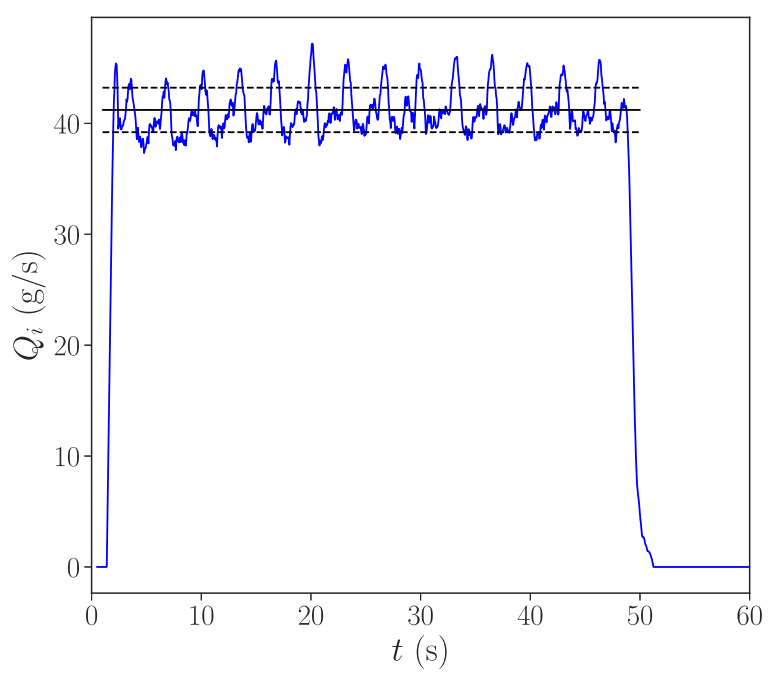

Fig. 2. Temporal evolution of the instantaneous mass flow rate $Q_{i}$ for $d_{p}=190 \mu \mathrm{m}, D=12 \mathrm{~mm}$ with obstacle $\left(D_{\text {obs }}=20 \mathrm{~mm}\right.$ and $h_{O}=5 \mathrm{~mm}$ ). The horizontal full line represents the mean flow rate, $Q$, whereas the dashed lines represent the standard deviation.

Table 1. Experimental parameters.

\begin{tabular}{ll}
\hline$D(\mathrm{~mm})$ & $12 ; 17.5 ; 24$ \\
$d_{p}(\mu \mathrm{m})$ & $190 ; 550 ; 1180 ; 4350$ \\
$D_{x}(\mathrm{~mm})$ & $0 ; 5 ; 10 ; 15 ; 20 ; 25 ; 30$ \\
$D_{y}(\mathrm{~mm})$ & $0 ; 5 ; 10 ; 15 ; 20 ; 25 ; 30$ \\
$h_{O}(\mathrm{~mm})$ & $0 ; 5 ; 10 ; 15 ; 20 ; 30 ; 40$ \\
$D_{\text {obs }}(\mathrm{mm})$ & $5 ; 10 ; 20 ; 40$ \\
\hline
\end{tabular}

\section{Effect of an obstacle on the discharge flow rate}

A first series of experiments was carried out with the configuration with an obstacle above the outlet (Fig. 1b). The obstacles have been positioned at different altitudes $h_{O}$ above the outlet and the mean flow rate $\mathrm{Q}$ was measured. For a constant outlet length $(D=12 \mathrm{~mm})$ and the smallest particles $\left(d_{p}=190 \mu \mathrm{m}\right)$, Figure 3 presents the mean flow rate versus the altitude $h_{O}$ of the obstacle for various obstacle diameters $D_{\text {obs }}$. On this figure, the red 


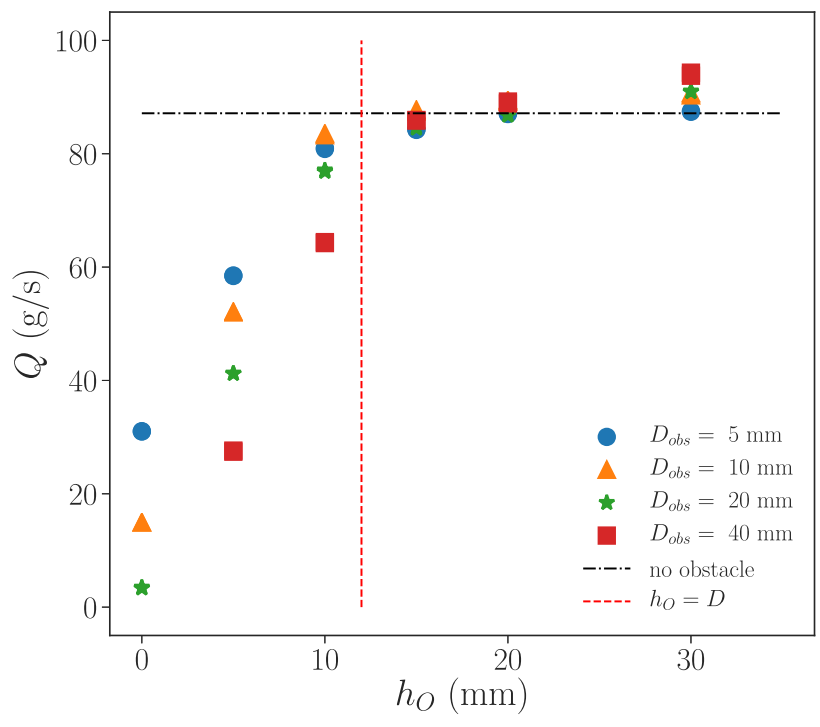

Fig. 3. Mass flow rate, $Q$, versus the obstacle altitude, $h_{O}$, for $d_{p}=190 \mu \mathrm{m}, D=12 \mathrm{~mm}$ and various obstacle diameters $D_{\text {obs }}$. The horizontal dotted-dashed line represents the flow rate without obstacle. The vertical dashed line represents the altitude where $h_{O}=D$.

dashed vertical line represents $h_{O}=D$ whereas the horizontal dotted dashed line represents the flow rate without obstacle. It is clear that the characteristic distance on which the obstacle modifies significantly the flow rate is for $h_{O}<D$. When $h_{O}$ becomes smaller then $D$, the mass flow rate decreases, and reaches the minimum value when the obstacle bottom is aligned with the bottom of the silo $\left(h_{O}=0\right)$. In this case, the mass flow rate depends of the obstacle diameter $D_{\text {obs }}$, the bigger the obstacle the smaller the flow rate. If $h_{O}>D$, the flow rate seems to stabilise and to reach the value obtained with no obstacle, whatever the obstacle diameter.

To validate this last observation, experiments were carried out with a flat bottom silo without obstacle (Fig. 1a) for all particle diameters. In Figure $4 \mathrm{a}$ and $\mathrm{b}$ we compare the results obtained for the discharge of a silo without obstacle (large symbols) and those obtained for a silo with an obstacle placed in the area $h_{O}>D$ (small symbols). The flow rate is made dimensionless using the particle diameter (Fig. 4a) or the outlet size (Fig. 4b) and is plotted versus the number of beads in the aperture $D / d_{p}$. In both graph all the data collapse on a unique curve which is well fitted by equation (1) with $C=1.15$, $\alpha=0.70$ and $\beta=0.05$. For small particles the Hagen law where $Q \propto D W \sqrt{g D}$ is recovered (see the dashed line in Fig. 4a). This result confirms that the obstacle placed above $h_{O}=D$ has negligible influence on the discharge flow rate, which is consistent with the idea that the discharge flow rate of a silo is determined in an accelerating zone of typical size $D$, situated just above the orifice. A perturbation of the flow outside this region does not affect the flow rate. The value of the fitting parameter are of the same order of magnitude as found for glass beads [15]. The small difference might be due to the physical properties of the beads as friction coefficients or roughness. This point is still under investigation.
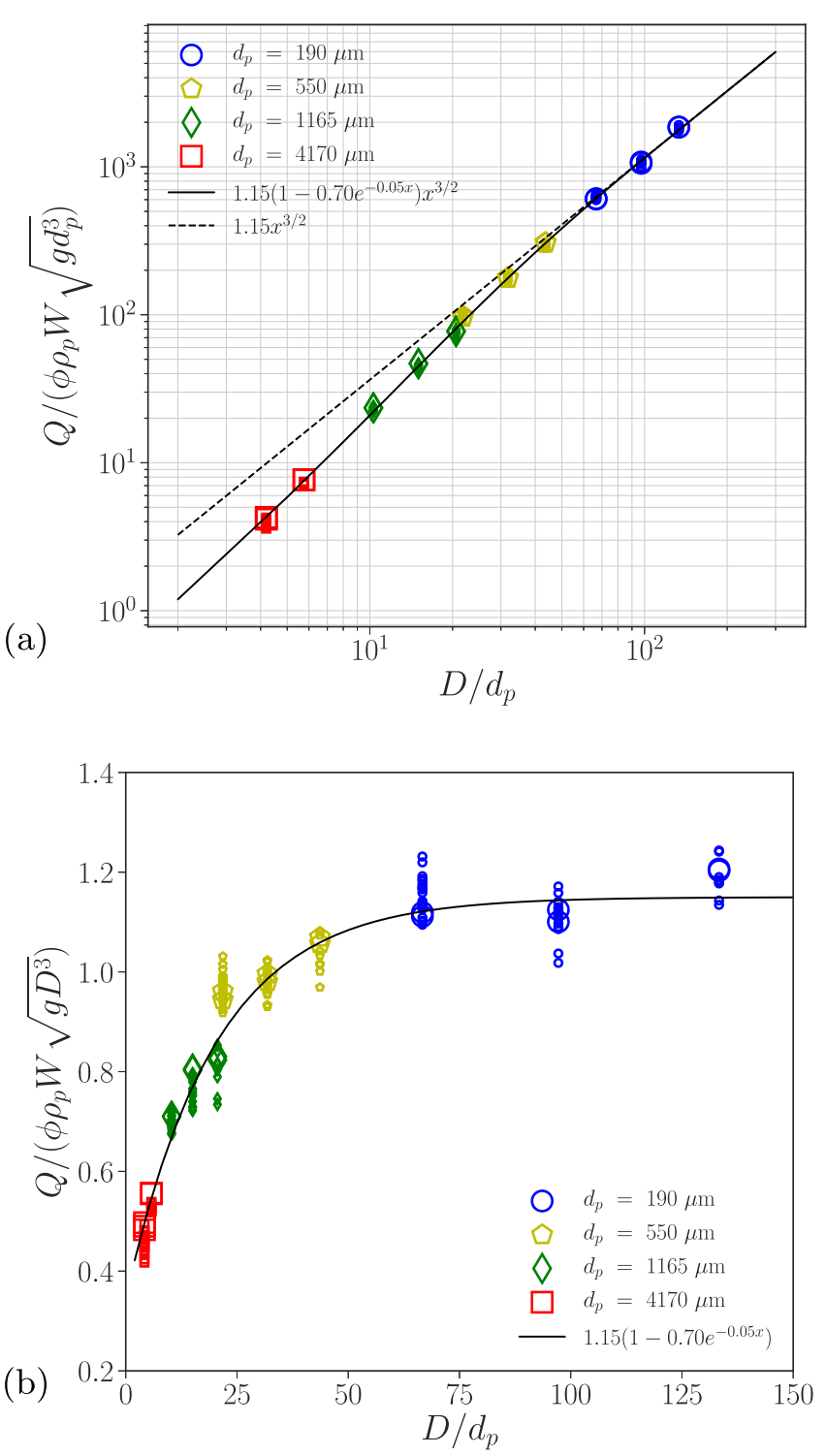

Fig. 4. Mass flow rate normalised by (a) $\rho_{p} \phi W \sqrt{g d_{p}^{3}}$ and (b) $\rho_{p} \phi W \sqrt{g D^{3}}$ as a function of $D / d_{p}$ for various particle diameters. The large symbols represent experiments without obstacle. The small symbols represent experiments with obstacle far from the outlet $h_{O}>D$. The full line represents equation (1) with $C=1.15, \alpha=0.7$ and $\beta=0.05$. The dashed lines represent the asymptotic regime when $D / d_{p} \gg 1$.

When $h_{O}$ becomes smaller than $D$, equation (1) is not valid anymore and the mass flow rate is controlled by an another length. Indeed the $\Pi$-theorem implies that the flow rate must scale like $\rho_{p} \sqrt{g \ell^{5}}$ (see [16] for more details) where $\ell$ is any combination of $D, W, h_{O}, D_{\text {obs }}$ and $d_{p}$. A geometrical analysis of the outlet area with an obstacle (Fig. 5a) points out that the outlet length $D$ is not the smallest length of the problem. We thus define a new outlet length $D_{\text {new }}$ which is a function of $D, h_{O}$ and $D_{\text {obs }}$ :

$$
D_{\text {new }}=\sqrt{\frac{D^{2}}{4}+\left(h_{O}+\frac{D_{\text {obs }}}{2}\right)^{2}}-\frac{D_{\text {obs }}}{2} .
$$


(a)
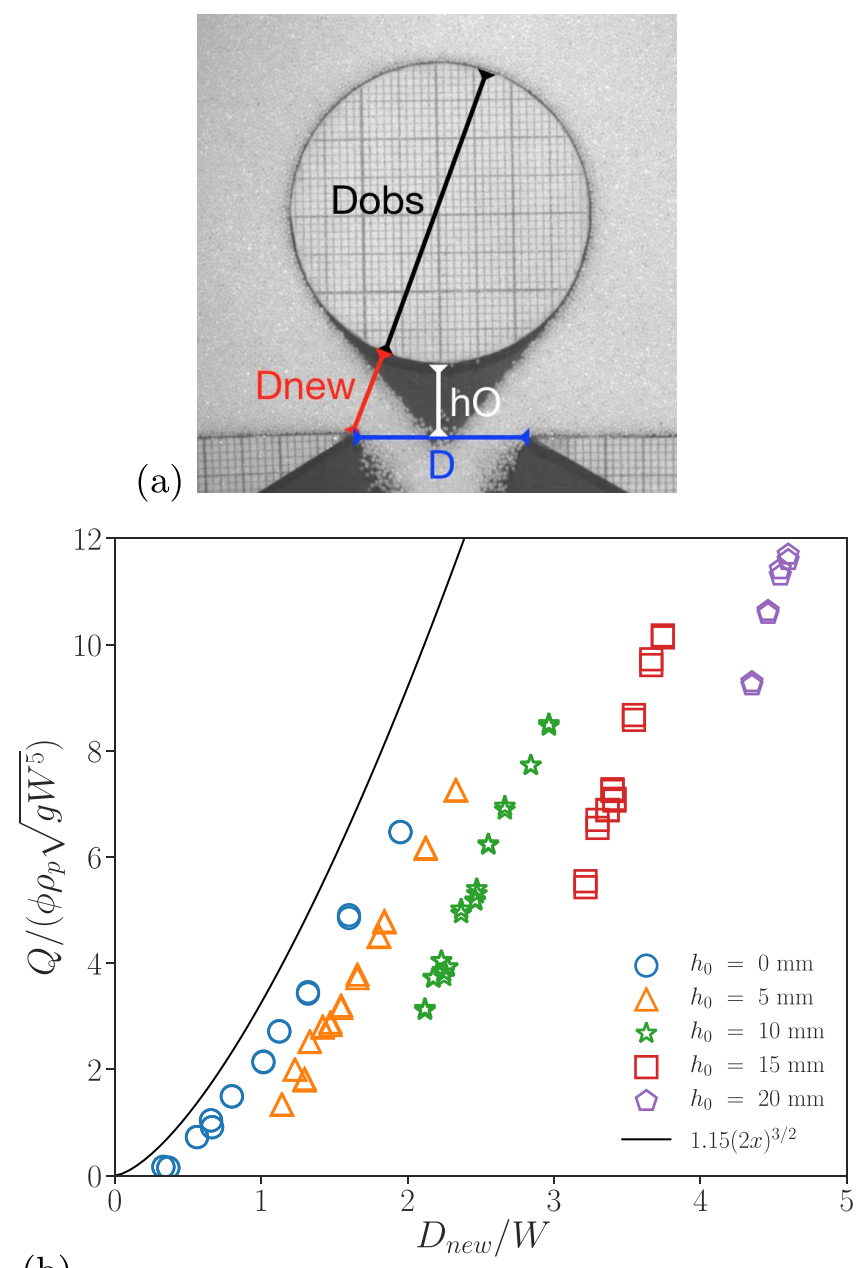

(b)

Fig. 5. (a) Definition of $D_{\text {new }}$ with in this snapshot $d_{p}=$ $550 \mu \mathrm{m}, D=24 \mathrm{~mm}, D_{\text {obs }}=40 \mathrm{~mm}$ and $h_{O}=10 \mathrm{~mm}$. (b) Mass flow rate $Q$ normalised by $\rho_{p} \phi \sqrt{g W^{5}}$ versus $D_{\text {new }} / W$ for all the experiments with $d_{p}=190 \mu \mathrm{m}$ and $h_{O} \leq D$. The full line represents the asymptotic regime of equation (1) when $D / d_{p} \gg 1$ with $C=1.15$ corresponding to the case without obstacle.

Figure $5 \mathrm{~b}$ presents the normalised mass flow rate $Q /\left(\rho_{p} \phi \sqrt{g W^{5}}\right)$ versus $D_{\text {new }}$ normalised by the silo thickness $W$ for the smallest particles $d_{p}=190 \mu \mathrm{m}$ and for various obstacle altitude with $h_{O} \leq D$. (For these small particles we expect no effect of the particle size $d_{p}$.) The full line represents the asymptotic regime of equation (1) when $D / d_{p} \gg 1$ with $C=1.15$ corresponding to the case without obstacle. We observe that the data do not collapse and are below the prediction of equation (1) which indicates that $D_{\text {new }}$ is not the relevant parameter.

The prediction of the mass flow rate in this configuration is complex and involves many parameters. However we observe that the obstacle separates the granular flow in two symmetrical arms (Fig. 5a) which merges in the zone around the outlet. Then to understand the parameters governing the flow rate, we propose to study the flow in one arm, but in a new and more simple configuration with an outlet in the right bottom corner (Fig. 1c).

\section{Discharge flow of a rectangular silo from a corner}

This configuration allows us to control precisely the outlet with only two parameters: the horizontal and vertical extensions of the aperture, respectively $D_{x}$ and $D_{y}$ (see Fig. 1c and Fig. 8b). We can then define a new outlet length $D_{\text {newc }}$ given by:

$$
D_{\text {newc }}=\left(D_{x}^{2}+D_{y}^{2}\right)^{1 / 2} .
$$

Figure $6 \mathrm{~b}$ presents the normalised mass flow rate versus $D_{\text {new }} / W$ for $d_{p}=190 \mu \mathrm{m}$ and for the two extreme positions $D_{y}=0$ (Fig. 6a) and $D_{x}=0$ (Fig. 6c). We first observe that $D_{x}$ and $D_{y}$ do not play the same role. For an orifice at the bottom, $D_{y}=0$, the flow rate is always at least 3 times higher than for a lateral orifice, $D_{x}=0$, and scales on $\left(D_{\text {new } c} / W\right)^{3 / 2}$ as predicted by equation (1) for $D_{\text {newc }} / d_{p} \gg 1$. To confirm this observation, experiments were carried out with all particle sizes in order to check the validity of the law. Results are presented on Figure 7. The normalised flow rate, using the particle diameter (Fig. 7a) or $D_{\text {newc }}=D_{x}$ (Fig. $\left.7 \mathrm{~b}\right)$ are in good agreement with equation (1) keeping the same $\alpha$ and $\beta$ than for the bottom orifice situated at the centre of the silo (Fig. 1a and Fig. 4) with the fitting parameter $C_{\text {new }}=1.41$ instead of $C=1.15$.

This difference of coefficient implies that for a bottom orifice the flow rate is higher at the border of the silo than at the centre. To understand this effect, we can use the sketch drawn in Figure 7c. If we consider an outlet at the centre of the silo, the velocity is zero at the border of the outlet and maximum at its centre. For an outlet at the border, if we first suppose that the vertical wall behaves as a free slip boundary then by symmetry the maximum velocity will be at the wall and the flow rate will correspond to half the flow rate of an orifice of size $2 D_{x}$. This leads to a coefficient $C_{\max }=\sqrt{2} C=1.62$ which is the maximum value which can be observed. In our experiment the measured value lies between $C$ and $C_{\max }$ which means that the granular media is sliding on the wall, as observed in [17], but not freely. The value of $C_{\text {new }}$ certainly depends on the wall friction coefficient, this will be explored in another study.

Considering now the case of a lateral orifice with $D_{x}=0$ (red square symbols in Fig. 6b), we observe that the flow rate is mainly linear with $D_{\text {newc }} / W$ (dashed line) which corresponds to the second regime described experimentally and numerically by Zhou et al. [16] for a silo discharge with a lateral orifice. This shows that the silo thickness is also an important parameter of control of the discharge flow from a corner, due to the friction on the front and rear wall. Some more experiments will be carried out with a larger silo for ceramic beads in order to change the value of $W$ and characterise the transition between the two regimes observed by Zhou et al. [16].

When $D_{x}$ and $D_{y}$ are non-zero values, all our results are in between these two regimes as we can see in Figure $8 \mathrm{~b}$ which presents the normalised mass flow rate $Q /\left(\rho_{p} \phi \sqrt{g W^{5}}\right)$ versus $D_{\text {newc }} / W$. This figure shows that 
(a)

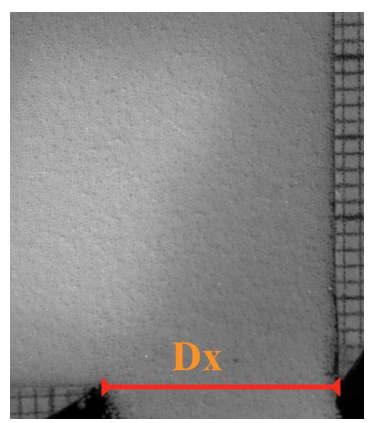

(b)

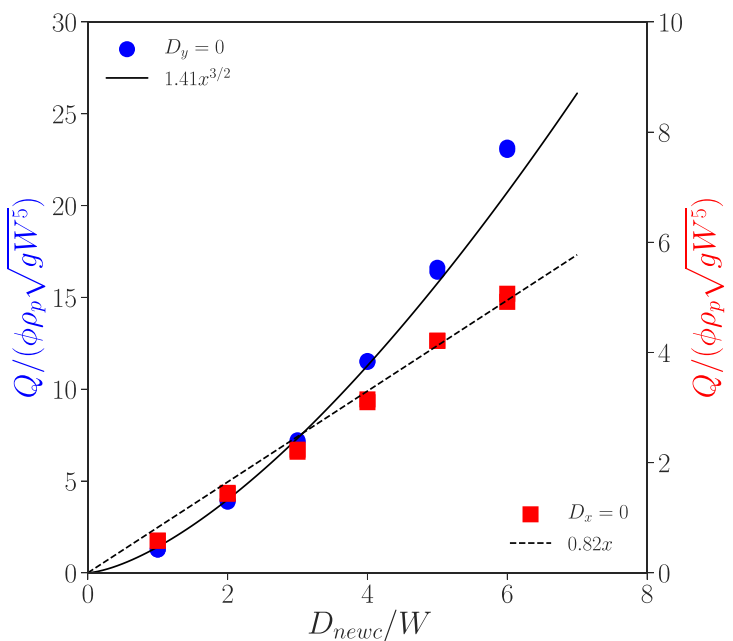

(c)

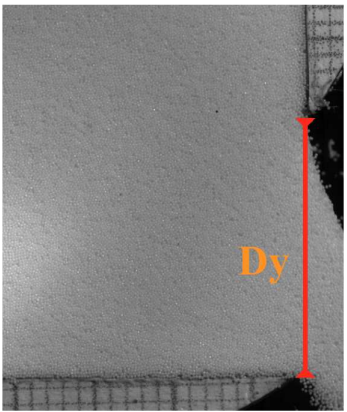

Fig. 6. (a) Configuration for $D_{y}=0$. (b) Mass flow rate $Q$ normalised by $\rho_{p} \phi \sqrt{g W^{5}}$ versus $D_{\text {newc }} / W$ for $d_{p}=190 \mu \mathrm{m}$ for a bottom orifice $\left(D_{y}=0\right)$ and a lateral orifice $\left(D_{x}=0\right)$. The full line represents the asymptotic regime of equation (1) when $D / d_{p} \gg 1$. The dashed line corresponds to the asymptotic regime of equation (2) when $D_{\text {newc }} / W \gg 1$. (c) Configuration for $D_{x}=0$

(a)

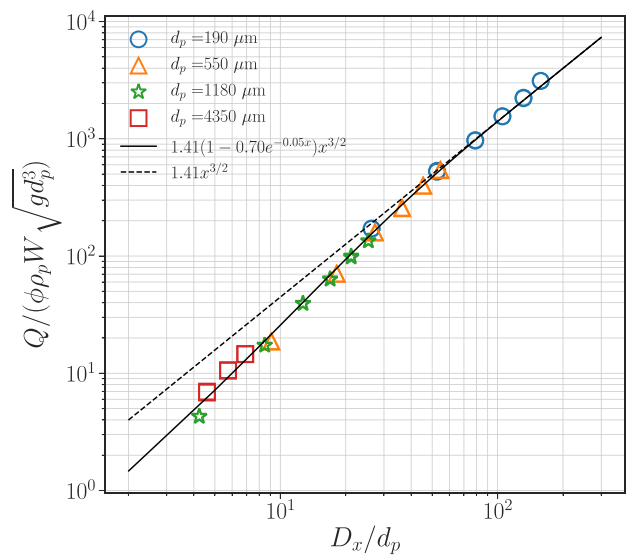

(b)

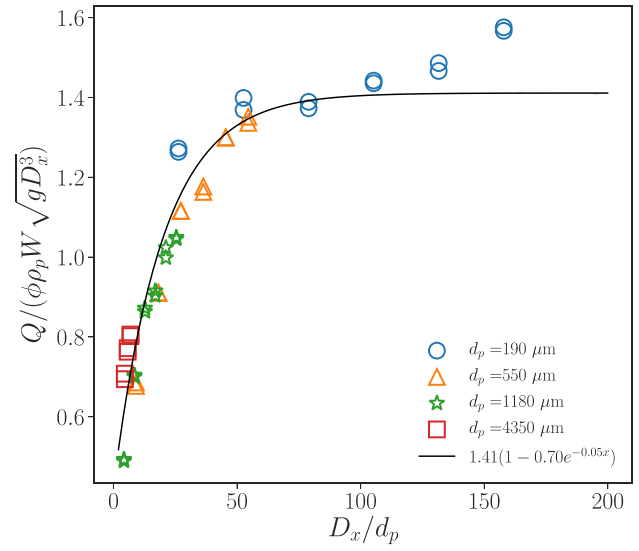

(c)

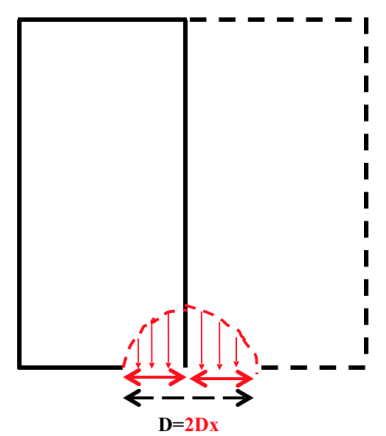

Fig. 7. Mass flow rate (a) normalised by $\rho_{p} \phi W \sqrt{g d_{p}^{3}}$ and (b) normalised by $\rho_{p} \phi W \sqrt{g D^{3}}$ as a function of $D_{\text {newc }} / d_{p}$ for various particle diameters and for a bottom orifice $D_{y}=0$. The full line represents equation (1) with $C=1.41, \alpha=0.7$ and $\beta=0.05$. The dashed lines represent the asymptotic regime when $D / d_{p} \gg 1$. (c) Sketch of the free slip boundary.

$D_{\text {newc }}$ is not the correct parameter to describe the discharge flow rate from a corner. However varying $D_{y}$ we observe the same trend than varying $h_{O}$ in the presence of an obstacle (Fig. 5b) which gives support to the analogy between the flow around an obstacle and the flow through a corner. Further work will be done experimentally (particle image velocimetry on the images and varying the thickness of the silo) and numerically (continuum simulations using a granular rheology) to better understand the physical process and the relevant parameters.

\section{Conclusion}

We have experimentally studied the discharge flow of ceramic beads from a rectangular silo in the presence of a cylindrical obstacle. We have shown that for an altitude higher than the orifice length the obstacle does not infer on the flow rate, while when situated in the zone of acceleration above the outlet the presence of the obstacle decreases significantly the flow rate. We have shown that the flow rate depends on all the parameters varied: the outlet size, the obstacle position and diameter, and possibly the silo thickness and the particle diameters. Using the minimum distance between the obstacle and the silo does not allow to scale the data. To simplify the problem we have experimentally studied the discharge flow of ceramic beads from a rectangular silo from a corner. We have shown that for a bottom orifice at the wall the flow rate obeys the same law than in the centre (Eq. (1)), with a slightly higher coefficient due to the boundary condition at the wall. For a lateral orifice we observe two regimes of flow (Eq. (2)) where for large aperture and narrow silo the flow is dominated by the parietal friction and depends on the silo thickness. For the corner, we observe that all 
(a)
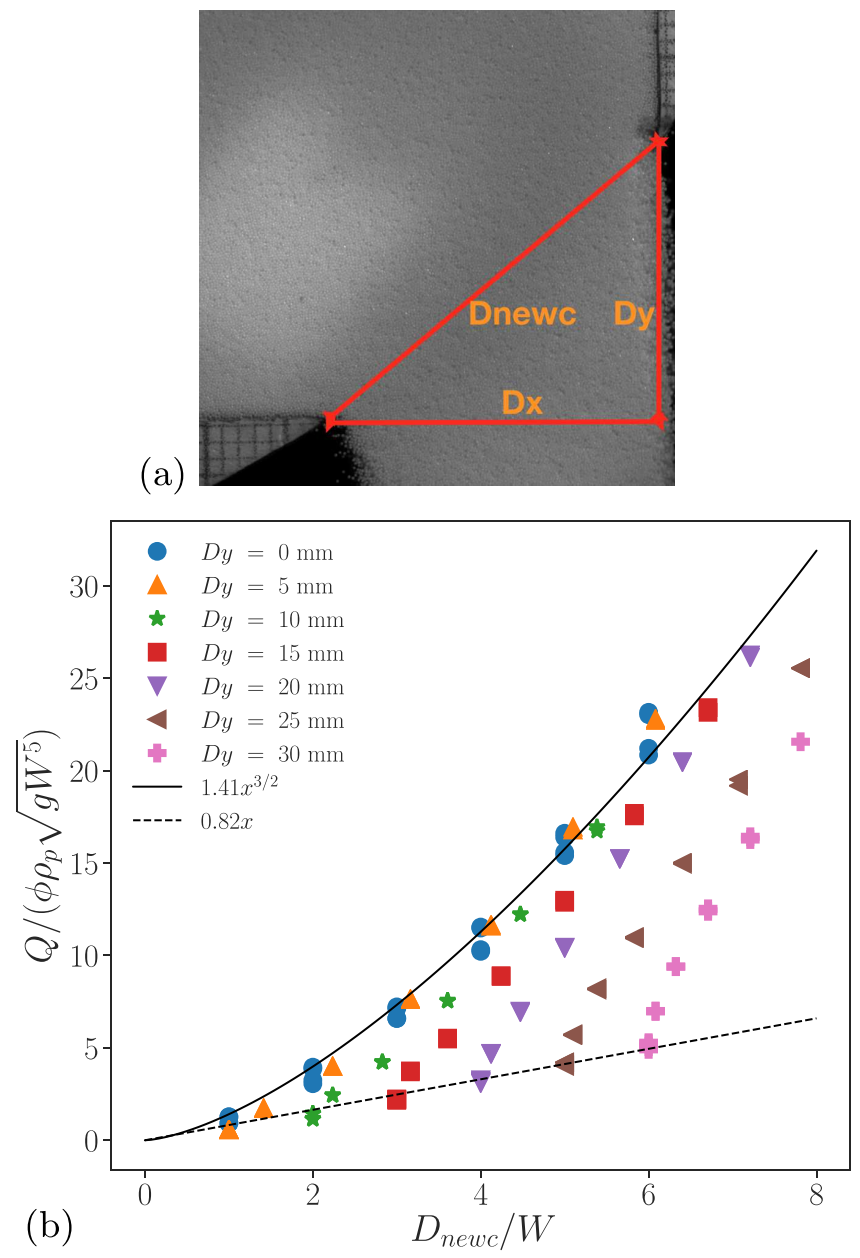

Fig. 8. (a) Definition of $D_{\text {newc }}$ with in this snapshot $d_{p}=$ $190 \mu \mathrm{m}, D_{x}=25 \mathrm{~mm}$ and $D_{y}=20 \mathrm{~mm}$. (b) Mass flow rate $Q$ normalised by $\rho_{p} \phi \sqrt{g W^{5}}$ versus $D_{\text {newc }} / W$ for all the experiments with $d_{p}=190 \mu \mathrm{m}$. The full line represents the asymptotic regime of equation (1) when $D / d_{p} \gg 1$ with $C=1.41$ (case $\left.D_{y}=0\right)$. The dashed line represents the asymptotic regime of equation (2) when $D_{\text {newc }} / W \gg 1$ (case $\left.D_{x}=0\right)$.

the data are in between these two cases, which suggest that the silo thickness is indeed a parameter which has to be taken into account in the discharge flow from a corner or in the presence of an obstacle. To better understand the physical process and the relevant parameters, future works will be devoted to the study of particle velocities using image processing and the thickness of the silo will be varied.

S. L. thanks Bechar University, Aix-Marseille University, IUSTI laboratory and Franco-Algerian program Profas B + for support. This work was undertaken under the auspices of the Labex MEC (ANR-10-LABX-0092) and of the A*MIDEX project (ANR-11-IDEX-0001-02), funded by the Investissements
d'Avenir program managed by the French National Research Agency (ANR).

\section{References}

[1] I. Zuriguel, A. Janda, A. Garcimartin, C. Lozano, R. Arevalo, D. Maza, Silo clogging reduction by the presence of an obstacle, Phys. Rev. Lett. 107, 278001 (2011)

[2] C. Lozano, A. Janda, A. Garcimartin, D. Maza, I. Zuriguel, Flow and clogging in a silo with an obstacle above the orifice, Phys. Rev. E 86, 031306 (2012)

[3] F. Alonso-Marroquin, S.I. Azeezullah, S.A. Galindo-Torres, L.M. Olsen-Kettle, Bottlenecks in granular flow: When does an obstacle increase the flow rate in an hourglass? Phys. Rev. E 85, 020301(R) (2012)

[4] J.R. Johanson, The use of flow corrective inserts in bins, J. Eng. for Ind. 2, 224-230 (1966)

[5] J.R. Johanson, The placement of inserts to correct flow in bins, Powder Technol. 1, 328-333 (1967/68)

[6] R.M. Nedderman, S.T. Davies, D.J. Horton, The flow of granular materials round obstacles, Powder Technol. 25, 215-223 (1980)

[7] U. Tüzün, R.M. Nedderman, Gravity flow of granular materials round obstacles-I: Investigation of the effects of inserts on flow patterns inside a silo, Chem. Eng. Sci 40, 325-336 (1985)

[8] S.C. Yang, S.S. Hsiau, The simulation and experimental study of granular materials discharged from a silo with the placement of inserts, Powder Technol. 120, 244 (2001)

[9] C.S. Chou, T.L. Yang, The effect of a flow corrective insert upon flow patterns and wall stresses in a two-dimensional bin-hopper, Adv. Powder Technol. 15, 567 (2004)

[10] J. Härtl, J.Y. Ooi, J.M. Rotter, M. Wsjcik, S. Ding, G.G. Enstad, The influence of a cone-in-cone insert on flow pattern and wall pressure in a full-scale silo, Chem. Eng. Res. Des. 86, 370 (2008)

[11] B.P. Tighe, M. Sperl, Pressure and motion of dry sand: translation of Hagen's paper from 1852, Granular Mater. 9, 141-144 (2007)

[12] W.A. Beverloo, H.A. Leniger, J.V. De Velde, The flow of granular solids through orifices, Chem. Eng. Sci. 15, 260$269(1961)$

[13] A. Janda, I. Zuriguel, D. Maza, Flow rate of particles through apertures obtained from self-similar density and velocity profiles, Phys. Rev. Lett. 108, 248001 (2012)

[14] S. Rubio-Largo, A. Janda, D. Maza, I. Zuriguel, R. Hildalgo, Disentangling the free-fall arch paradox in silo discharge, Phys. Rev. Lett. 114, 238002 (2015)

[15] M. Benyamine, M. Djermane, B. Dalloz-Dubrujeaud, P. Aussillous, Discharge flow of a bidisperse granular media from a silo, Phys. Rev. E 90, 032201 (2014)

[16] Y. Zhou, P.-Y. Lagrée, S. Popinet, P. Ruyer, P. Aussillous, Experiments on, and discrete and continuum simulations of, the discharge of granular media from silos with a lateral orifice, J. Fluid Mech. 829, 459-485 (2017)

[17] R. Maiti, G. Das, P.K. Das, Experiments on eccentric granular discharge from a quasi-two-dimensional silo, Powder Technol. 301, 1054-1066 (2016)

Cite this article as: S. Laidaoui, P. Aussillous, M. Djermane, B. Dalloz-Dubrujeaud, Discharge flow of granular media from rectangular silos: role of an obstacle and modelling by an orifice at the corner, Mechanics \& Industry 21, 516 (2020) 